\title{
REMOTE SENSING OF AEROSOLS BY SYNERGY OF CALIOP AND MODIS
}

\author{
Rei Kudo $^{1 *}$, Tomoaki Nishizawa ${ }^{2}$, Akiko Higurashi $^{2}$, and Eiji Oikawa ${ }^{3}$ \\ ${ }^{1}$ Meteorological Research Institute, JMA, Japan, *reikudo@mri-jma.go.jp \\ ${ }^{2}$ National Institute for Environmental Studies, Japan \\ ${ }^{3}$ National Institute of Information and Communication Technology
}

\begin{abstract}
For the monitoring of the global 3-D distribution of aerosol components, we developed the method to retrieve the vertical profiles of water-soluble, light absorbing carbonaceous, dust, and sea salt particles by the synergy of CALIOP and MODIS data. The aerosol product from the synergistic method is expected to be better than the individual products of CALIOP and MODIS. We applied the method to the biomass-burning event in Africa and the dust event in West Asia. The reasonable results were obtained; the much amount of the water-soluble and light absorbing carbonaceous particles were estimated in the biomass-burning event, and the dust particles were estimated in the dust event.
\end{abstract}

\section{INTRODUCTION}

Aerosols have significant impacts on the Earth's radiation budget. It is essential to investigate the spatial and temporal variability of the microphysical and optical properties of aerosols.

The remote sensing from the space has a role to monitor the aerosols in the global scale. The satellite-borne passive sensor, such as MODIS, provides the global distribution of the columnar properties of the aerosols [1]. The active sensor onboard the satellite, such as CALIOP, can capture the vertical profile of aerosols [2]. A synergistic remote sensing using both the passive and active sensors makes up for each other's weak points and have an ability to provide the better result of aerosols. For example, the aerosol vertical profile is assumed in the retrieval using the passive sensor although the radiance observed by the passive sensor depends on the vertical profile. The active sensor can provide the information of the vertical profile.

We are developing a new method to retrieve the vertical profiles of aerosol components by the synergy of CALIOP and MODIS. The data and the retrieval algorithm are described in Sect. 2. Two case studies are shown in Sect. 3. Finally, the results are summarized in Sect. 4.

\section{METHODOLOGY}

\subsection{Data}

The CALIOP data used in this study is the attenuated backscatter at 532 and $1064 \mathrm{~nm}$, the depolarization ratio at $532 \mathrm{~nm}$, and the vertical feature mask of the CALIPSO Lidar Level 1B and 2 Products Version 3. The vertical resolution is $120 \mathrm{~m}$. The horizontal resolution is $1 \mathrm{~km}$. The MODIS data is the radiances in band $1(620-670$ $\mathrm{nm})$ and $2(841-876 \mathrm{~nm})$ of Level 1B Calibrated Radiances (MYD021KM). The horizontal resolution is $1 \mathrm{~km}$. We selected the radiance scattered from a point near the CALIOP observation and used it for the retrieval.

We used the auxiliary data in the forward modeling of the aerosol physical and optical properties, the CALIOP and MODIS data. The data is the pressure, the temperature, the relative humidity, and the surface wind speed on the ocean derived from the GEOS-5 meteorological data product. In calculating the radiance on the land, the surface albedo of the MODIS global albedo product (MCD43C3) was used.

\subsection{Retrieval algorithm}

We assumed four aerosol components, watersoluble (WS), light absorbing carbonaceous (LAC), dust (DS), and sea salt (SS). These are the major components in the atmosphere and have different particle sizes and light absorption characteristics. The vertical profiles of the extinction coefficients of four components are retrieved by simultaneously optimizing their vertical profiles of the dry volume concentrations, and their vertically mean dry mode-radii to the CALIOP and MODIS data. The optimization is 
conducted by the method developed by Kudo et al. [3]. This method optimizes the parameters by minimizing the objective function defined as

$$
\begin{aligned}
f(\mathbf{x})= & \frac{1}{2}\left(\mathbf{y}^{o b s}-\mathbf{y}(\mathbf{x})\right)^{T}\left(\mathbf{W}^{2}\right)^{-1}\left(\mathbf{y}^{o b s}-\mathbf{y}(\mathbf{x})\right) \\
& +\frac{1}{2} \mathbf{y}_{a}(\mathbf{x})^{T}\left(\mathbf{W}_{a}^{2}\right)^{-1} \mathbf{y}_{a}(\mathbf{x})
\end{aligned}
$$

where vector $\mathbf{y}_{\mathrm{obs}}$ is the CALIOP and MODIS observation data, vector $\mathbf{x}$ comprises the dry volume concentrations and the vertically mean dry mode-radii of WS, LAC, DS, and SS, vector $\mathbf{y}(\mathbf{x})$ is the calculated values by the forward model, matrix $\mathbf{W}$ is the standard errors in the observations, vector $\mathbf{y}_{\mathrm{a}}$ is the constraints to $\mathbf{x}$, and matrix $\mathbf{W}_{\mathrm{a}}$ is parameters to determine the strength of the constraints.

Our developed forward model consists of three parts. In the first part, the aerosol optical properties are calculated from the dry volume concentrations and the vertically mean dry moderadii. Other two parts are the calculations of the CALIOP and MODIS data.

The particle shape of WS, LAC, and SS were assumed to be spherical. The DS was assumed to be the randomly oriented spheroids [4]. The LAC was assumed to be a core-gray shell model, which is an internal mixture model, and its optical properties are better than those of the core-shell model and the homogeneous internal mixture model [5]. We defined the WS as the shell around a core of LAC, and the shell-core ratio was fixed by 1.5 . This lies among the values observed in the A-FORCE aircraft campaign [6].

The size distribution of each component was assumed to be a lognormal function, and the values of the standard deviations were given from the database of OPAC [7]. The vertically mean dry mode-radii of WS, LAC, and DS are parameters to be optimized. We assumed that the dry mode-radii of WS and LAC are same. The dry mode-radius of SS was parameterized by the surface wind speed on the ocean [8].

The refractive index of WS and SS were given form the OPAC [8]. The refractive index of LAC was given form the measurements reported in [9]. The refractive index of DS is given from the result of the ADEC project [10].
Before calculating the optical properties from the size distribution and the refractive index, the volumes and mode radii of WS, SS, and the shell of LAC are increased by the hygroscopic growth depending on the relative humidity of the ambient condition. Their growth factors and the refractive index for the particle mixed with the water were given from the database of OPAC [8].

The optical properties of WS and SS were calculated using Mie theory. The optical properties of LAC were calculated using the stratified sphere Mie code [11]. The optical properties of DS are calculated by using the data table developed by [4].

The attenuated backscatter coefficients for co- and cross polarization are calculated by the lidar equation. The radiances are calculated by PSTAR, a vector radiative transfer model developed by [12].

We constrained the range that the values of $\mathbf{x}$ can be by some methods. The smoothness of the vertical profiles of WS, DS, and SS are given by

$$
\begin{aligned}
y_{a}(\mathbf{x}) & =\ln \left(V_{j}\left(z_{i-1}\right)\right) \\
& -2 \ln \left(V_{j}\left(z_{i}\right)\right)+\ln \left(V_{j}\left(z_{i+1}\right)\right)
\end{aligned}
$$

where $V$ is the dry volume concentration at the altitude $\mathrm{z}$ for $j=\mathrm{WS}, \mathrm{LAC}, \mathrm{DS}$, and SS.

We assumed that the vertical profile of LAC has a similar shape to that of WS and introduced the shape constraint for LAC by,

$$
\begin{aligned}
y_{a}(\mathbf{x}) & =\ln \left(V_{L A C}\left(z_{i}\right) / V_{L A C}\left(z_{i+1}\right)\right) \\
& -\ln \left(V_{W S}\left(z_{i}\right) / V_{W S}\left(z_{i+1}\right)\right)
\end{aligned} .
$$

In general, it is considered that the aerosol optical depth (AOD) of LAC is smaller than that of WS. This inequality was introduced by using the penalty function,

$$
y_{a}(\mathbf{x})=\left\{\begin{array}{cc}
-\ln \left(1-\tau_{L A C} / \tau_{W S}\right) & \text { for } \tau_{L A C}<\tau_{W S} \\
+\infty & \text { otherwise }
\end{array},\right.
$$

where $\tau$ is the aerosol optical depth.

When the AOD of LAC approaches to the AOD of WS, the penalty function rapidly increases. 
The retrieval of the dry mode radii of WS, LAC, and DS is very challenging. In the numerous tests of our algorithm using the simulated and actual data of CALIOP and MODIS, the mode-radii can be retrieved from the noiseless data of CALIOP and MODIS. The retrieval of the mode-radii was influenced by the noise of CALIOP data. For the stable retrieval, the a priori values of the dry mode-radii for WS, LAC, and DS are given by,

$$
y_{a}(\mathbf{x})=\ln \left(r_{j}\right)-\ln \left(r_{a, j}\right),
$$

where $r$ is the dry mode-radii, and $r_{a}$ is a priori values for $j=\mathrm{WS}, \mathrm{LAC}$, and DS.

Based on the above mentioned forward model and constraints, the dry volume concentrations and the vertically mean dry mode-radii of WS, LAC, DS, and SS are optimized to the CALIOP and MODIS data. Finally, the extinction coefficients of each component are output.

\section{RESULTS}

Two case studies of the biomass-burning and dust events are presented. Figures 1 and 2 show the case of the biomass-burning event in Africa. The CALIPSO satellite passed by the region where the fires are active (Figure 1). The retrieved extinction coefficients are characterized by the large values of WS and LAC (Figure 2). In general, the large amount of the fine particles and the strong light absorbing property are observed in the biomassburning event.

Figures 3 and 4 show the case of the dust event in West Asia. The CALIPSO passed by the desert region (Figure 3). The retrieved extinction coefficient was characterized by the large value of WS and DS.

\section{CONCLUSIONS}

We developed the synergistic method to retrieve the vertical profiles of aerosol components from the CALIOP and MODIS data. The products are the extinction coefficients of WS, LAC, DS, and SS. Two case studies for the biomass-burning and dust events showed the consistent results with each scene. We will apply the method to the longterm data of CALIOP and MODIS to investigate the global 3-D distributions of aerosol components.
We plan to apply our method to the high spectral resolution lidar and the imaging spectral radiometer onboard the EarthCARE satellite (Earth Clouds, Aerosols and Radiation Explorer) [13]. The lidar ratio derived from the high spectral resolution lidar is expected to improve the retrieval of LAC because the lidar ratio significantly depends on the light absorption particles.

\section{ACKNOWLEDGEMENTS}

This work was supported by the Japan Society for the Promotion of Science KAKENHI grant no. $15 \mathrm{H} 02808$.

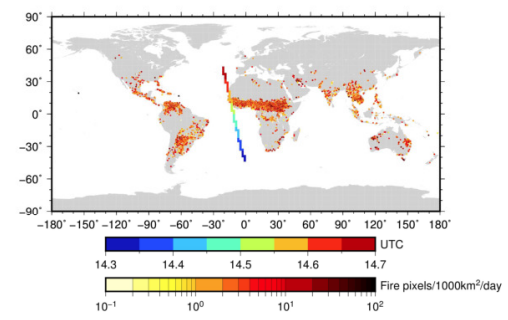

Figure 1 The orbit of CALPSO in 29 Jan, 2010, and the fire activity from $25 \mathrm{Jan}$ to $2 \mathrm{Feb}, 2010$. The color of the orbit is time (UTC). The fire activity is based on data of MODIS active-fire product.
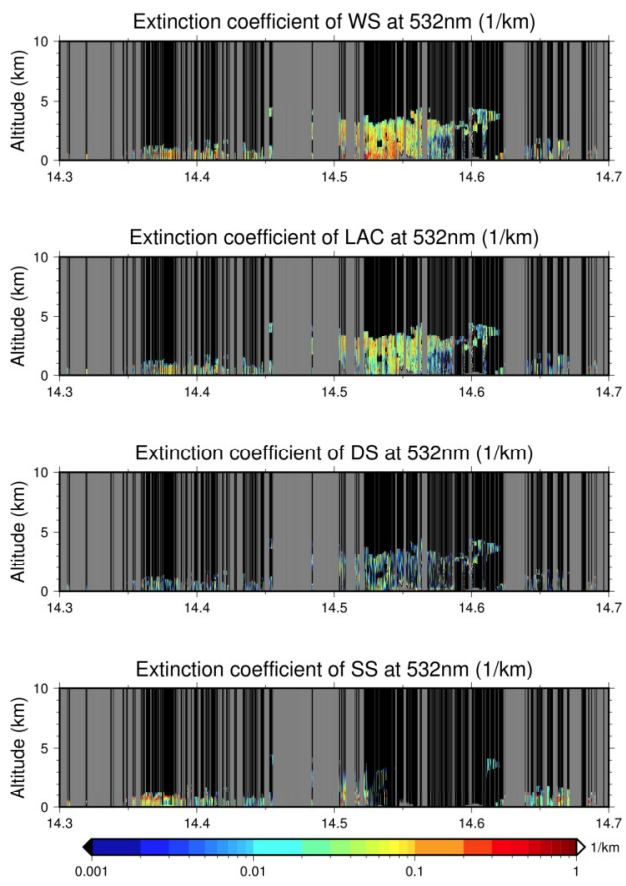

Figure 2 Retrieved extinction coefficients of WS, LAC, $D S$, and SS in the biomass-burning event. The gray 
region indicates the cases that include clouds. Our method cannot be applied to such cases.

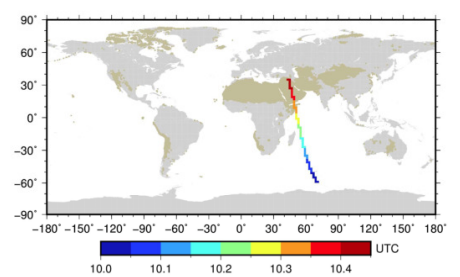

Figure 3 The orbit of CALPSO in 30 Jan, 2010. The color of the orbit is time (UTC). The ochre region indicates barren or sparsely vegetated region.
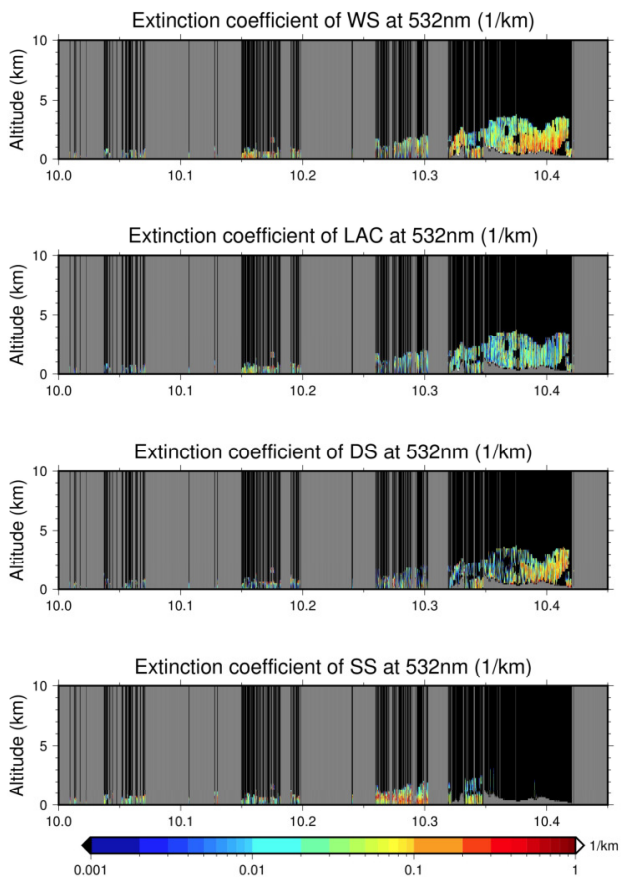

Figure 4 Retrieved extinction coefficients of WS, LAC, $D S$, and SS in the dust event. The gray region indicates the cases that include clouds. Our method cannot be applied to such cases.

\section{References}

[1] Higurashi, A, and Nakajima, T., 1999: Development of a two-channel aerosol retrieval algorithm on a global scale using NOAA AVHRR, J. Atmos. Sci. 56, 924-941.

[2] Winker, D. M., et al., 2009: Overview of the CALIPSO mission and CALIOP data processing algorithms, J. Atmos. Oceanic Technol. 26, 2310-2323.

[3] Kudo, R., et al., 2016: Vertical profiles of aerosol optical properties and the solar heating rate estimated by combining sky radiometer and lidar measurements, Atmos. Meas. Tech. 9, 3223-3243.

[4] Dubovik, O., et al., 2006: Application of spheroid models to account for aerosol particle nonsphericity in remote sensing of desert dust, J. Geophys. Res. 111, D11208, doi:10.1029/2005JD006619.

[5] Kahnert, M., et al., 2013: Models for integrated and differential scattering optical properties of encapsulated light absorbing carbon aggregates, Opt. Express 21, 7, 7974-7993.

[6] Matsui, H., et al., 2013: Development and validation of a black carbon missing state resolved three-dimensional model: Aging process and radiative impact, J. Geophys. Res. 118, 2304-2326.

[7] Hess, M., et al., 1999: Optical properties of aerosols and clouds: The software package OPAC, Bull. Amer. Metor. Soc. 79, 831-844.

[8] Erickson, D. J., and Duce, R. A., 1988: On the global flux of atmosphere sea salt, J. Geophys. Res. 93, 14079-14088.

[9] Chang, H., and Charalampopoulos, T. T., 1990: Determination of the wavelength dependence of refractive indices of flame soot, Proc. R. Soc. Lond. A 430, 577-591.

[10] Aoki, T., et al., 2005: Sensitivity experiments of direct radiative forcing caused by mineral dust simulated with a chemical transport model, J. Meteor. Soc. Jpn. 83A, 315-331.

[11] Toon, O. B., and Ackermann, T. P., 1981: Algorithms for the calculation of scattering by stratified spheres, Appl. Opt. 20, 3657-3660.

[12] Ota, Y., et al., 2010: Matrix formulations of radiative transfer including the polarization effect in a coupled atmosphere-ocean system, $J$. Quant. Spect. Rad. Trans. 111, 6, 878-894.

[13] Illingworth, A. J., et al., 2015: The EARTHCARE satellite: the next step forward in global measurements of clouds, aerosols, precipitation, and radiation, Bull. Am. Meteor. Soc., 96, 1311-1332. 